\title{
Actualización en diagnóstico y tratamiento del ataque cerebrovascular isquémico agudo
} Diagnosis and Treatment of Acute Ischemic Stroke: An Update

Fecha de recepción: 11/11/2018 | Fecha de aprobación: 25/02/2019

\author{
Carolina García Alfonso \\ Pontificia Universidad Javeriana, Colombia \\ Andrea Martínez Reyes \\ Pontificia Universidad Javeriana, Colombia \\ VALENTINA GARCÍA \\ Pontificia Universidad Javeriana, Colombia \\ Andrés Ricaurte-Fajardo \\ Pontificia Universidad Javeriana, Colombia \\ ISABEL TORRES \\ Pontificia Universidad Javeriana, Colombia \\ Juliana Coral \\ Pontificia Universidad Javeriana, Colombia
}

a Correspondencia: acgarcia@husi.org.co

\section{RESUMEN}

El ataque cerebrovascular (ACV) agudo es la segunda causa de muerte en el mundo y genera costos elevados en su tratamiento y recuperación, así como un gran impacto socioeconómico, pues es la principal causa de discapacidad a largo plazo. Si bien la tomografía axial computarizada (TAC) cerebral simple sigue siendo la imagen recomendada por las guías internacionales para la evaluación inicial y toma de decisiones sobre el manejo del paciente con sospecha de ACV, en los últimos años ha habido extraordinarios avances en el diagnóstico oportuno y temprano del ACV con nuevas herramientas que van desde la estandarización de la angiotomografía cerebral como uno de los estudios principales en el enfoque inicial hasta el uso de técnicas de perfusión por tomografía y resonancia magnética (RM) cerebral, que permiten establecer el núcleo del infarto y el área circundante potencialmente salvable, por lo que es posible ofrecer terapias que brindan al paciente funcionalidad y calidad de vida a mediano y largo plazo. Teniendo en cuenta lo anterior, es vital actualizar los métodos diagnósticos actuales disponibles en el país y las distintas terapias disponibles, según sea el caso de cada paciente, para el ACV isquémico agudo, con un enfoque clínico práctico y aplicable al escenario actual de salud en Colombia.

Palabras clave

ataque cerebrovascular agudo; ictus; infarto cerebral; terapia trombolítica; trombólisis.

\begin{abstract}
Acute stroke is the second cause of death worldwide, generating high costs in its treatment and recovery, as well as a great socioeconomic impact, being the main cause of long-term disability. Although, the simple computerized tomography (CT) scan of the brain is still the neuroimage recommended by the international guidelines for the initial evaluation
\end{abstract}


and decision making on the management of the patient with suspected stroke, recent years have witnessed extraordinary advances in the timely and early diagnosis of stroke, with new tools, from the standardization of cerebral angiotomography as an initial study, to perfusion techniques which allows to establish the nucleus of the area and the potentially salvageable surrounding area, as well as, therapies that give the patient support to the functionality and quality of life in the medium and long term. Taking into account the above, it is of vital importance an update on the available methods and on the different options available according to the case of each patient for acute stroke, with a practical clinical approach and applicable real health scenario in Colombia.

Keywords

acute cerebrovascular accident; brain infarction; stroke; thrombolysis; thrombolytic therapy.

\section{Introducción}

La enfermedad cerebrovascular es un término jerárquicamente amplio. Es un síndrome que incluye un grupo de enfermedades heterogéneas con un punto en común: una alteración en la vasculatura del sistema nervioso central, que lleva a un desequilibrio entre el aporte de oxígeno y los requerimientos de oxígeno, cuya consecuencia es una disfunción focal del tejido cerebral $(1,2)$.

El accidente cerebrovascular (ACV), por otra parte, se refiere a la naturaleza de la lesión, y se clasifica en dos grandes grupos: isquémico y hemorrágico. El ACV isquémico agudo se genera por oclusión de un vaso arterial e implica daños permanentes por isquemia; no obstante, si la oclusión es transitoria y se autorresuelve, se presentarán manifestaciones momentáneas, lo cual haría referencia a un ataque isquémico transitorio, que se define como un episodio de déficit neurológico focal por isquemia cerebral, de menos de 60 minutos de duración, completa resolución posterior, y sin cambios en las neuroimágenes (2).

Por otro lado, el ACV de origen hemorrágico es la ruptura de un vaso sanguíneo que lleva a una acumulación hemática, ya sea dentro del parénquima cerebral o en el espacio subaracnoideo (2).

El ACV agudo es una emergencia neurológica frecuente, con 17 millones de casos anuales en el mundo $(3,4)$, y es la segunda causa de muerte después de la enfermedad coronaria $(3,5)$, con 6,5 millones de pérdidas al año. Representa una alta carga de morbilidad y genera altos costos en atención médica inicial, tratamiento y rehabilitación en los distintos sistemas de salud del mundo. Por eso, en los últimos años se han desarrollado múltiples terapias de manejo que buscan disminuir la mortalidad, brindando funcionalidad y calidad de vida.

Es vital dar a conocer las nuevas herramientas disponibles para el diagnóstico oportuno, desde la estandarización de la angiotomografía cerebral como estudio inicial hasta técnicas de perfusión por tomografía y resonancia magnética (RM) cerebral, que permiten establecer el núcleo del infarto y el área circundante potencialmente salvable.

El objetivo de esta revisión es presentar una actualización sobre los métodos diagnósticos actuales y las distintas terapias disponibles según sea el caso de cada paciente, para el $\mathrm{ACV}$ isquémico agudo, con un enfoque clínico práctico, ordenado y aplicable al escenario actual de salud en Colombia.

\section{Epidemiología}

El ACV isquémico agudo constituye la quinta causa de muerte en Estados Unidos y la cuarta en el Reino Unido, la cual varía según la raza y la etnia, pues es casi dos veces más alto en la población afroamericana. En Estados Unidos se registran anualmente 610.000 casos nuevos y 185.000 casos recurrentes, de los cuales hasta el $87 \%$ es de origen isquémico y generan costos de aproximadamente 34 billones de dólares por año (6). Es la causa más importante de discapacidad grave a largo plazo con consecuencias catastróficas para el paciente y su familia. Se prevé que el ACV representará el 6,2\% de la carga total de la enfermedad en los países desarrollados en el 2020.

Los datos estadísticos en Colombia son escasos..El estudio Epineuro, publicado en el 2003, determinó la prevalencia de ocho problemas neurológicos frecuentes y encontró 
que para el ACV la prevalencia fue del 19,9\%, más frecuente en mayores de 50 años y mujeres. Adicionalmente, se cuenta con una base de datos que muestra las tromboembolectomías y trombólisis llevadas a cabo en Colombia entre 2011 y 2015 e identifica que Bogotá es la ciudad con el mayor número de procedimientos en el país (6).

\section{Fisiopatología}

\section{Autorregulación cerebral}

El flujo sanguíneo cerebral (FSC) está determinado por la resistencia vascular cerebral, directamente relacionada con su diámetro. Es el proceso por medio del cual el FSC se mantiene constante a pesar de variaciones en la presión de perfusión. El mantenimiento del FSC ocurre dentro de un rango de presión arterial media de 60 a $150 \mathrm{~mm} \mathrm{Hg}$. Fuera de este rango, el cerebro no puede compensar los cambios en la presión de perfusión, ya que aumenta el riesgo de isquemia a bajas presiones y edema a altas presiones (7).

El ACV isquémico disminuye el FSC y la presión de perfusión cerebral. En el estadio I, el FSC se mantiene constante gracias a la dilatación máxima de arterias y arteriolas, lo que produce un aumento compensatorio en el volumen sanguíneo cerebral. En el estadio II, cuando se agota la vasodilatación máxima, la fracción de extracción de oxígeno se incrementa para mantener la oxigenación y el metabolismo del tejido cerebral. En el estadio III, cuando en el núcleo isquémico se supera el rango autorregulatorio disminuye el volumen y el FSC hasta que la circulación colateral falla, ocasionando muerte celular $(8,9,10,11)$.

\section{Cascada isquémica}

La isquemia genera una cascada de eventos que conducen a muerte neuronal; incluye disminución en la producción de adenosín trifosfato (ATP); cambios en las concentraciones de sodio, potasio y calcio; aumento de lactato; acidosis; acumulación de radicales libres; acumulación intracelular de agua, y estimulación persistente de los receptores de glutamato (12).

Las cinasas de proteínas dependientes de calcio, la fosfolipasa A2, el óxido nítrico sintetasa $(\mathrm{SON})$, las endonucleasas y las proteasas se activan, acumulando sodio y calcio intraneural que revierte la absorción del glutamato en los astrocitos; a la vez que aumenta la excitotoxicidad y la activación de fosfolipasas que lesionan la membrana celular, proteasas que fragmentan el ADN y el citoesqueleto, lipooxigenasas, ciclooxigenasas, oxidasa de xantinas y SON, que aumentan los radicales libres citotóxicos, ácidos grasos libres y derivados del ácido araquidónico $(7,13,14)$ (figura 1).

\section{Figura 1}

Generación de radicales libres en la isquemia

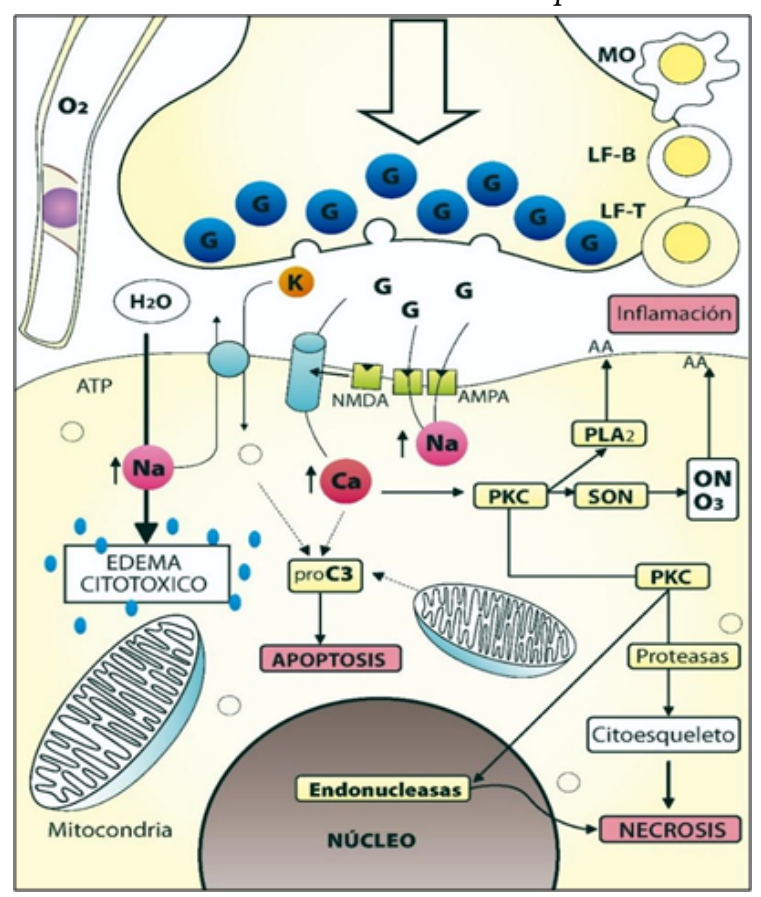

Fuente: adaptada de Tratamiento actual del ACV isquémico (ACV) agudo. Univ Méd (Bogotá). 2008;49(4):467-98.

La activación del receptor N-metil-Daspartato (NMDA) lleva a la producción de óxido nítrico. La actividad de la SON y la cantidad de óxido nítrico aumentan después de la exposición a la hipoxia (15). El óxido nítrico sintetasa $(\mathrm{SON})$ neuronal y el SON inducible 
generan mayores cantidades de óxido nítrico, al lesionar componentes celulares; además, puede reaccionar con el anión superóxido, produciendo peroxinitrito, que fragmenta cadenas simples del ADN y activa apoptosis (16).

\section{Necrosis y apoptosis}

La muerte celular después de la isquemia ocurre por necrosis o por apoptosis. La inflamación aumenta el FSC a la región isquémica, que puede suministrar glucosa y oxígeno a las células; sin embargo, este aumento del FSC libera calcio, que resulta en aumento del daño tisular $(7,17)$. La necrosis predomina en el centro del infarto y la apoptosis en el área de penumbra isquémica $(18,19)$ (figura 2).

\section{Figura 2}

Activación de la apoptosis en isquemia

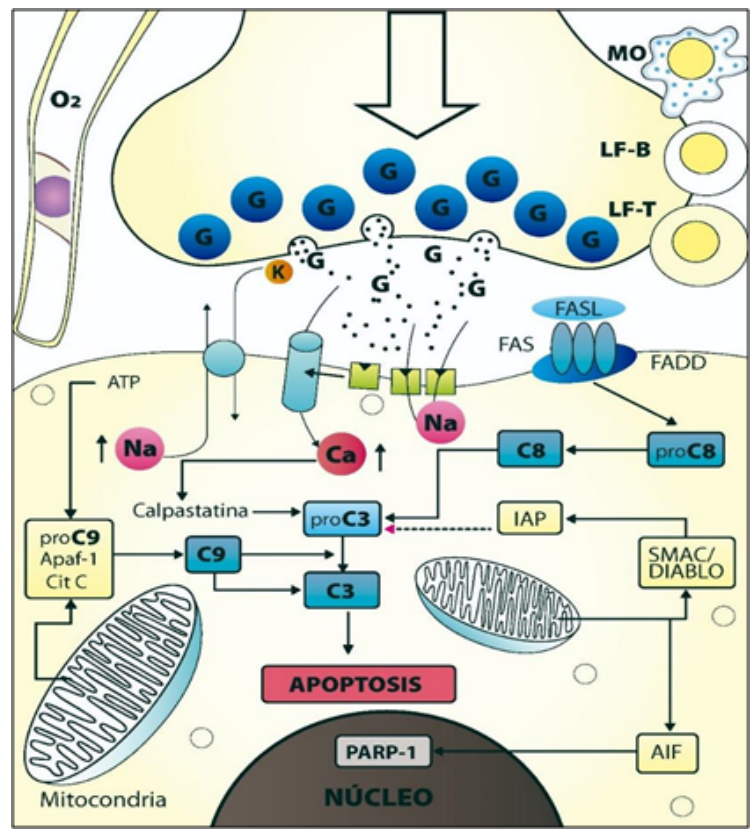

Fuente: adaptada de Tratamiento actual del ACV isquémico (ACV) agudo. Univ Méd (Bogotá). 2008;49(4):467-98.

La necrosis se acompaña de edema celular, lesión del tejido circundante, lisis de la membrana celular y lesión de los organelos. La circulación colateral produce energía suficiente para permitir la expresión de proteínas que median la apoptosis. Los fragmentos celulares generados conforman el "cuerpo apoptótico" (13). La apoptosis se genera mediante una serie de cambios que incluye las caspasas 1, 3, 8, 9 y 11, que activan factores que destruyen proteínas clave para la supervivencia (20).

\section{Penumbra isquémica}

Es el área de tejido que rodea el centro del infarto, con afectación funcional pero potencialmente viable. La isquemia incluye áreas que se recuperan espontáneamente, denominadas áreas de oligohemia benigna, y áreas que pueden progresar a cambios irreversibles, denominadas áreas de penumbra. La progresión a infarto depende del grado de circulación colateral, la duración de la lesión y el metabolismo celular. La oligohemia benigna se asocia a FSC mayor de $17 \mathrm{ml}$ por minuto por cada $100 \mathrm{~g}$ de tejido; la penumbra isquémica a valores entre 10 y $17 \mathrm{ml}$ por minuto por cada $100 \mathrm{~g}$ de tejido; y el core del infarto, a menos de $10 \mathrm{ml}$ por minuto por cada $100 \mathrm{~g}$ de tejido (21) (figura 3).

Figura 3

Áreas de isquemia

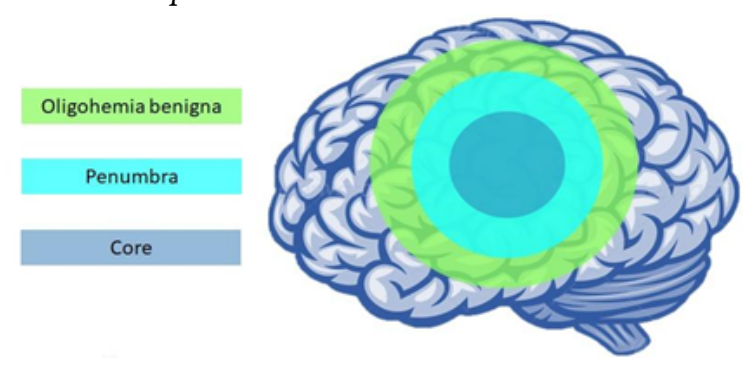

Edema cerebral

Aproximadamente, el $10 \%$ de los ACV isquémicos se clasifican como malignos, debido a la presencia de edema cerebral, aumento de presión intracraneal y herniación cerebral (22). Los dos mecanismos fisiopatológicos del edema son citotóxico y vasogénico. En el edema citotóxico, la isquemia por estrés oxidativo genera expresión de canales no selectivos, como el receptor tipo 1 para la sulfonilurea y el 
NCca-ATP, que ingresan masivamente sodio a la célula. La apertura de estos canales se efectúa 2 a 3 horas después del inicio de la lesión isquémica y se desencadena por la disminución del ATP. El resultado es la acumulación de agua intraneuronal $(23,24)$. El edema vasogénico es causado por el aumento de la permeabilidad de la barrera hematoencefálica, permitiendo que macromoléculas entren en el espacio extracelular, lo que aumenta el volumen de fluido a este nivel (23).

\section{Etiología}

Existen 5 categorías etiológicas del ACV según la escala de TOAST (25), y su categorización ha probado ser benéfica al optimizar el tratamiento específico de cada paciente.

1. Enfermedad aterotrombóticaaterosclerótica de gran vaso: la isquemia es generalmente de tamaño medio o grande, de topografía cortical o subcortical y localización vertebrobasilar o carotídea. Debe cumplir uno de los dos criterios:

a) Aterosclerosis con estenosis: estenosis $>50 \%$ de diámetro luminal u oclusión de la arteria extracraneal correspondiente o de la arteria intracraneal de gran calibre.

b) Aterosclerosis sin estenosis: estenosis $<50 \%$ en ausencia de otra etiología y con al menos dos de los siguientes factores de riesgo: $>50$ años, hipertensión arterial, diabetes mellitus, dislipidemia o tabaquismo.

2. Cardioembolismo: isquemia de tamaño medio o grande, de topografía cortical en la que existe alguna cardiopatía de características embolígenas.

3. Enfermedad oclusiva de pequeño vaso infarto lacunar: isquemia de pequeño tamaño $<1,5 \mathrm{~cm}$ de diámetro en el territorio de una arteria perforante cerebral que puede ocasionar un síndrome lacunar.

4. Otras causas: isquemia de tamaño variable de localización cortical o subcortical, en territorio carotídeo o vertebrobasilar, en un paciente en el que se han descartado las tres anteriores. Se puede producir por enfermedades sistémicas, alteraciones metabólicas, alteraciones de la coagulación, disección arterial, displasia fibromuscular, migraña, malformación arteriovenosa, etc.

5. De origen indeterminado: por estudio incompleto, por más de una etiología $\mathrm{o}$ por origen desconocido y estudio completo $(26,27)$.

\section{Cuadro clínico}

Los signos y síntomas se manifiestan según la localización y extensión de la lesión (28). Los principales territorios vasculares que pueden verse alterados son:

Circulación anterior: arteria carótida interna, arteria cerebral media y anterior.

Arteria cerebral anterior: presentará hemiparesia e hipoestesia contralateral de predominio crural, disartria, incontinencia urinaria, apatía, abulia, desinhibición y mutismo acinético en caso de daño bilateral.

Arteria cerebral media en su porción más proximal (M1) presentará hemiplejia e hipoestesia contralateral, hemianopsia homónima, desviación forzada de la mirada, alteración del estado de conciencia y afasia si se afecta el hemisferio dominante. Las porciones M2-M3 se presentarán con hemiparesia e hipoestesia contralateral, disartria, afasia si se afecta el hemisferio dominante, y hemiamopsia homónima en compromiso de M2. Si el daño es en la porción M4, presentará los mismos signos y síntomas, pero de forma menos severa, y presentará más afectación de funciones corticales como el lenguaje, así como disgrafia, discalculia, agrafoestesia, apraxias o debutar con crisis. 
Circulación posterior: arteria cerebral posterior, arteria basilar y arteria vertebral.

Arteria cerebral posterior: afectación del campo visual contralateral, agnosia visual, o ceguera cortical o crisis visuales.

Territorio vertebrobasilar: pueden presentar compromiso cerebeloso o troncoencefálico de acuerdo con la arteria afectada. Existe daño de la punta de la basilar, que se presentará con compromiso del estado de conciencia, alteraciones pupilares $u$ oculomotoras, cerebelosas, y compromiso motor de las cuatro extremidades, que en caso de no ser identificado y tratado, puede llevar al paciente a la muerte en pocas horas.

En la evaluación inicial se utiliza la escala del National Institute of Health Stroke Scale (NIHSS), para evaluar la severidad del cuadro clínico (18) (tabla 1).

\section{Tabla 1}

\section{Escala de NIHSS para ACV}

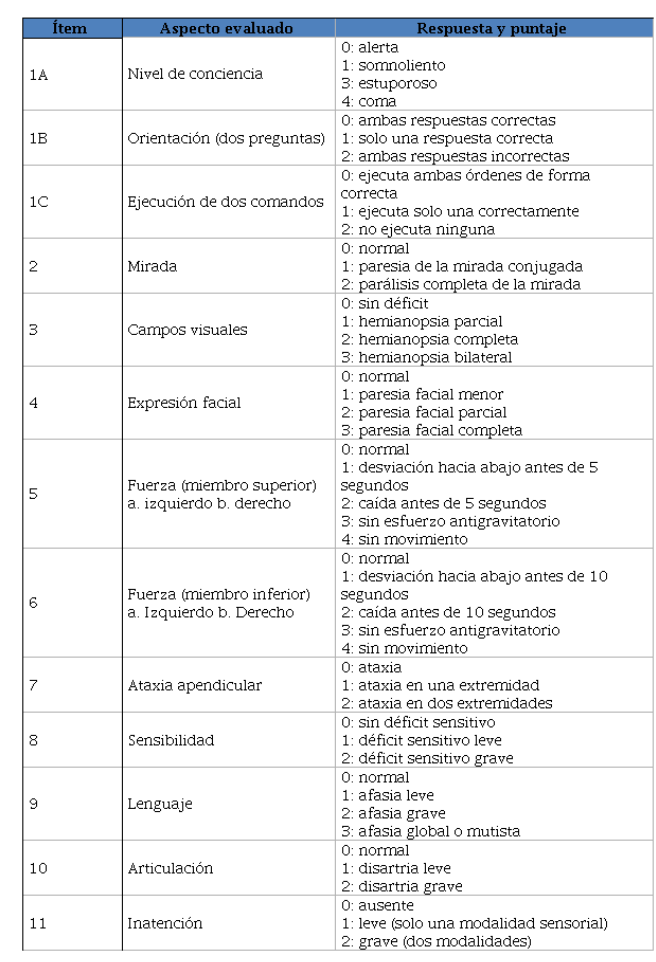

\section{Ayudas diagnósticas}

El diagnóstico del ACV isquémico agudo es clínico y los estudios imagenológicos se realizan con el fin de detectar hemorragia, evaluar el grado de lesión e identificar el territorio vascular afectado (29).

Dentro de los estudios imagenológicos que se exponen a continuación habrá mayor o menor disponibilidad de acuerdo con el centro clínico, el acceso a una unidad de ACV y la ciudad; así, la mayoría de estudios descritos están disponibles en ciudades principales del país, como Bogotá, Cali, Medellín, entre otras, y en lugares de bajo acceso se dispone de TAC cerebral simple, con opción de remisión a ciudades intermedias para realización de RM cerebral.

La tomografía axial computarizada (TAC) cerebral simple es la imagen recomendada por la Asociación Americana del Corazón (AHA) para la evaluación inicial y toma de decisiones sobre el manejo del paciente con sospecha de ACV, ya que la TAC es ampliamente disponible, tiene una alta sensibilidad y es relativamente rápida (30). Se recomienda su toma en los primeros 20 minutos de llegada al centro médico con el objetivo de diferenciar el ACV isquémico del hemorrágico, ya que este último contraindicaría el tratamiento con rtPA $(31,32)$.

La TAC simple permite calcular el Alberta Stroke Program Early Computed Tomography Score (ASPECTS), una escala cuantitativa (figura 4) para medir signos tempranos de isquemia cerebral (33). Para calcularlo se utilizan dos cortes axiales: el primero en los ganglios basales y el segundo en los ventrículos laterales y se divide el territorio de la arteria cerebral media (ACM) en diez regiones:

En el primer corte se debe valorar el núcleo caudado (C), lenticular (L), rodilla de la cápsula interna, brazo posterior (IC) y corteza insular (I). En cuanto a los territorios de la arteria cerebral media, se debe valorar la corteza anterior de la ACM (M1), la corteza lateral adyacente al ribete insular (M2) y la corteza posterior de la ACM (M3), es decir, siete áreas.

En el segundo corte se debe valorar el territorio anterior de la ACM (M4), el territorio lateral de 
la ACM (M5) y el territorio posterior de la ACM (M6), es decir, tres áreas.

\section{Figura 4}

ASPECTS. A). Territorios vasculares M1, M2, M3, cabeza de núcleo caudado (C), insula (I), núcleo lenticular (L). B). Territorios vasculares M4, M5, M6.
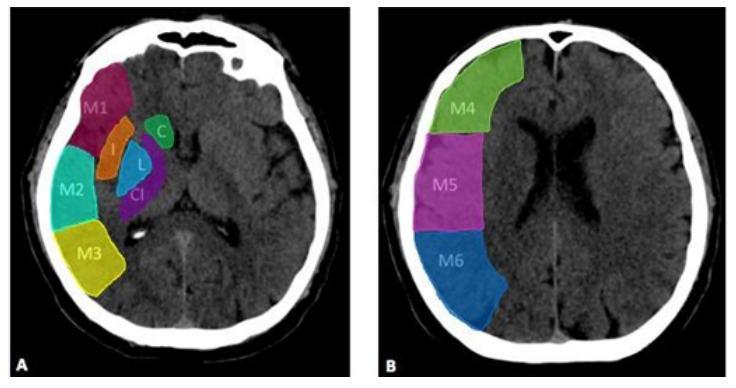

Lesiones hipodensas en alguna de esas 10 áreas resta un punto en la escala. Un puntaje de 10 implica un estudio normal y un puntaje de 0 indica afectación de todo el territorio de la ACM. El puntaje mínimo aceptado para ofrecer terapia trombolítica es de 7, un puntaje menor se relaciona con menor beneficio terapéutico (34).

En la TAC también se pueden observar otros elementos como cambios isquémicos tempranos dados por hipodensidades leves del parénquima con pérdida de la diferenciación de las sustancias gris y blanca. Los infartos antiguos que se observan como hipodensidades bien definidas del parénquima y la hiperdensidad de una arteria se asocia con oclusión trombótica de grandes vasos (30).

La angiografía por tomografía computarizada (angio-TAC) de cerebro y vasos de cuello requiere medio de contraste yodado endovenoso, que permite evaluar la anatomía vascular arterial. Es útil para detectar áreas de oclusión o estenosis y para identificar enfermedad vascular extracraneana $(31,34)$. La angio-TAC también es útil para caracterizar la morfología del trombo, ya que la longitud de este es uno de los factores predictores del desenlace funcional del paciente (35). Se ha demostrado que la angio-TAC es confiable para la evaluación de grandes vasos intracraneales, pues la AHA lo recomienda para pacientes candidatos a terapia endovascular (32).
En cuanto a la circulación colateral, algunos estudios han mostrado que la presencia de buenas colaterales se asocia con mejor respuesta a la trombólisis, la terapia endovascular y la reducción del volumen del core isquémico (36); otros estudios apoyan que los pacientes con pobres colaterales están predispuestos a complicaciones hemorrágicas y muerte después de la terapia endovascular (37). Sin embargo, un reciente estudio en pacientes llevados a terapia endovascular con ventana mayor a 6 horas mostró que la presencia de buenas colaterales en angio-TAC solo se asoció con un volumen del core isquémico más pequeño y una reducción en su crecimiento; pero no influyó en desenlaces como independencia funcional medida por Rankin, ni en el éxito de la terapia endovascular o reducción en las complicaciones hemorrágicas o la muerte (38). Por lo anterior, si bien la circulación colateral puede proveer un flujo sanguíneo crucial en los pacientes con ACV (39), no debe ser un criterio único a la hora de incluir o excluir pacientes para realización de terapia trombolítica o endovascular.

La TAC cerebral por perfusión es la imagen de elección para evaluar el área de penumbra isquémica. Requiere la administración de medio de contraste y muestra imágenes del FSC, el volumen sanguíneo cerebral (VSC) y el tiempo de tránsito medio (TTM) (31).

VSC: es el volumen total de sangre que contiene una determinada zona cerebral y se mide en milímetros de sangre por 100 gramos de tejido cerebral $(\mathrm{ml} / 100 \mathrm{~g})(8,34)$.

FSC: es el volumen de sangre que atraviesa una determinada zona cerebral por unidad de tiempo, medido en mililitros de sangre por 100 gramos de tejido por minuto $(\mathrm{ml} / 100 \mathrm{~g} / \mathrm{min})$ $(8,34)$.

Tiempos de circulación medidos en segundos, dentro de los que se incluyen: el TTM y el tiempo máximo (Tmáx), este último —cuyo valor normal es 0 - refleja el suministro normal de sangre al tejido sin demora $(8,34)$.

El TTM marca las áreas isquémicas y también incluye áreas limítrofes; es el parámetro más sensible para detectar la disminución de perfusión cerebral. El FSC delimita el área de 
isquemia crítica y el VSC delimita el área de necrosis ya instaurada. Los mapas de VSC y FSC generalmente muestran áreas de anormalidad más pequeñas que TTM, por lo tanto, son más específicos para las áreas de isquemia e infarto (40).

El core en un TAC por perfusión está definido como el área que tiene un FSC menor al 30\% del identificado en el hemisferio sano. El Tmáx mayor a 6 segundos muestra una zona isquémica del parénquima, pero aún no necrótica. Por esto, para definir el área de penumbra se usa la técnica de mismatch, definida como la diferencia entre la zona de necrosis y la de isquemia. Es decir, la zona de penumbra resulta de restar el core del área con Tmáx mayor a 6 segundos $(41,42)$.

Los estudios DAWN (DWI or CTP Assessment with Clinical Mismatch in the Triage of Wake-Up and Late Presenting Strokes Undergoing Neurointervention with Trevo) (41) y DEFUSE 3 (Endovascular Therapy Following Imaging Evaluation for Ischemic Stroke) (42) han demostrado la factibilidad de terapia endovascular con ventana de 6 a 24 horas, en pacientes con oclusión de gran vaso y neuroimágenes con evidencia penumbra isquémica. En dichos estudios, en pacientes elegibles, esta terapia influye en términos de discapacidad a los 90 días. La AHA recomienda imágenes de perfusión en pacientes con las consideraciones mencionadas en la tabla 2 (32).

\section{Tabla 2}

Criterios mismatch usados en los estudios DAWN y DEFUSE 3

\begin{tabular}{|c|c|}
\hline DAWN & DEFUSE 3 \\
\hline $\begin{array}{l}\text { Mismatch entre la gravedad del déficit } \\
\text { clínico y el tamaño del infarto temprano } \\
\text { en las imágenes }\end{array}$ & M Mismatch imagenológico \\
\hline $\begin{array}{l}- \text { Core isquémico (DWI en IRM o FSC } \\
\text { relativo en TAC por perfusión) } \\
\bullet \geq 80 \text { años, con NIHSS } \geq 10 \text { y core } \\
\text { isquémico } \\
-<21 \mathrm{ml} \\
-<80 \text { años, con NIHSS } \geq 10 \text { y core } \\
\text { isquémico }<31 \mathrm{ml} \\
-<80 \text { años, con NIHSS } \geq 20 \text { y core } \\
\text { isquémico } 31 \text { a } 51 \mathrm{ml}\end{array}$ & $\begin{array}{l}\text { - Core isquémico (DWI en IRM o FSC } \\
\text { relativo en TAC por perfusión) } \\
\text { - HipoperfuSión crítica (Tmáx }>6 \\
\text { segundos en imagen de perfusión por } \\
\text { TAC o IRM) } \\
\text { - Core isquémico }<70 \mathrm{ml} \\
\text { - Mismatch ratio } \geq 1,8 \\
\text { - Mismatch en volumen } \geq 15 \mathrm{ml}\end{array}$ \\
\hline
\end{tabular}

Resonancia magnética (RM) cerebral: dentro de las secuencias de RM convencional, la secuencia diffusion-weighted imaging (DWI) es útil para identificar cambios isquémicos tempranos, al detectar infarto hasta en un 95\% de las ocasiones. Una lesión isquémica aguda se verá de alta señal en el DWI, y se debe corresponder con una lesión de baja señal, en la misma localización, en el mapa de apparent diffusion coefficient (ADC). El tamaño del core es determinante en el pronóstico de estos pacientes. A mayor volumen de infarto, aumenta la probabilidad de desenlaces desfavorables. La RM cerebral no es un estudio de rutina para la evaluación inicial, ya que puede tardar mucho tiempo en completarse y tiene menor disponibilidad que la TAC (31).

En RM, el mismatch se realiza al comparar las imágenes potenciadas por perfusión (PWI) y el DWI. El área que muestra anomalías tanto de difusión como de perfusión representa un tejido infartado; mientras que el área que muestra solo anomalías de perfusión y tiene una difusión normal corresponde con el área de penumbra(43). En los ACV de tiempo indeterminado, las secuencias DWI, ADC y FLAIR precisan el tiempo de evolución de la lesión; imágenes de alta señal en DWI sin correspondencia en el FLAIR implican un tiempo menor de 4,5 horas con una sensibilidad del $62 \%$ y una especificidad del 78\% (8) (figura 5).

\section{Figura 5}

Imágenes de IRM cerebral. A) FLAIR. Lesión hiperintensa gangliobasal izquierda. B) DWI. Lesión hiperintensa con restricción de señal gangliobasal izquierda. C) ADC. Imagen de baja señal en mapa de ADC gangliobasal izquierda que se corresponde con localización de imágenes $A$ y $B$
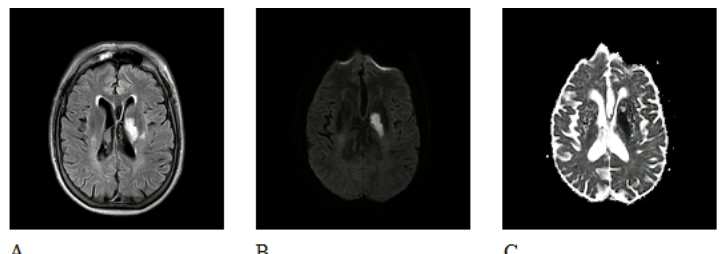

La angiografía por RM cerebral es útil para detectar oclusión o estenosis de la circulación intra- y extracraneal. Puede realizarse con contraste o sin este, aunque se prefiere el uso de contraste, por su mayor calidad de imagen; sin embargo, no es superior a la angio-TAC $(31,43)$.

La panangiografía cerebral consiste en la inserción de un catéter en la arteria femoral o 
braquial, que se dirige hasta los vasos cerebrales y se inyecta medio de contraste con toma de imágenes secuenciales para observar su paso por los vasos sanguíneos (parenquimograma). El uso de catéter hace posible el objetivo terapéutico del procedimiento, por medio de colocación de stent o trombectomía aspirativa (44).

Los pacientes candidatos a terapia endovascular deben cumplir los siguientes criterios (32):

1. Rankin previo menor a 2.

2. Edad mayor a 18 años.

3. NIHSS mayor a 8 .

4. ASPECTS mayor a 6.

5. Inicio de la intervención dentro de las primeras 6 horas de los síntomas.

La escala Thrombolysis in Cerebral Infarction (TICI) (33) evalúa los hallazgos angiográficos después del tratamiento intraarterial. Califica la revascularización/reperfusión posterior al procedimiento (8). Los puntajes son:

0 : sin flujo anterógrado.

1: flujo que es posterior a la oclusión, pero sin reperfusión tisular.

2a: reperfusión tisular parcial en $<66 \%$ del territorio de la arteria ocluida.

2b: reperfusión tisular completa del territorio de la arteria ocluida pero enlentecida respecto a la arteria homóloga contralateral.

3: reperfusión tisular completa y sin retraso.

Se ha demostrado una reducción significativa de la mortalidad en TICI 2 b y 3 , comparado con puntajes de TICI más bajos (12,8\% versus $39,1 \%$, respectivamente). Por lo tanto, se considera un TICI $2 \mathrm{~b}$ o 3 como un resultado angiográfico exitoso (45).

\section{Tratamiento}

Medidas generales y de soporte

Se recomienda el soporte de la vía aérea y asistencia ventilatoria como parte del manejo en pacientes con $\mathrm{ACV}$, que presenten alteración del estado de conciencia o disfunción bulbar que afecte la vía aérea (nivel I, clase C).
Además, se recomienda lograr saturaciones de oxígeno mayores a 94\%, aun si esto implica el uso de oxígeno suplementario (nivel I, clase C). La temperatura $>38{ }^{\circ} \mathrm{C}$ debe tratarse con antipiréticos (nivel I, clase C). La hiperglucemia persistente durante las primeras 24 horas posteriores a un ACV se asocia con un peor desenlace. Se recomiendan niveles de entre 140 y $180 \mathrm{mg} / \mathrm{dL}$ y evitar la hipoglucemia (nivel IIa), la cual debe tratarse cuando sea $<60 \mathrm{mg} / \mathrm{dL}$ (nivel I, clase C). Se deben usar antihipertensivos cuando las cifras de tensión arterial sean iguales o mayores a 220/120 mm Hg, sin descensos menores al $15 \%$ en las primeras 24 horas. Los pacientes candidatos a terapias de reperfusión deben mantener una presión arterial menor a 185/110 mm Hg (nivel I, clase B), y los pacientes que ya han sido llevados a terapia de reperfusión deben mantener una tensión menor a 180/105 $\mathrm{mm} \mathrm{Hg}$, durante las primeras 24 horas después del tratamiento $(1,32)$.

\section{Terapia trombolítica}

Las terapias de reperfusión en ACV isquémico agudo son:

Trombólisis intravenosa (IV).

Trombólisis intraarterial (IA).

Trombectomía mecánica.

Ventanas de reperfusión para pacientes con ACV isquémico agudo: el tiempo de ventana para terapias de reperfusión se empieza a contar a partir de la "última vez que fue visto normal" o cuando un testigo lo cuantifica.

\section{Trombólisis IV con alteplase (tabla 3)}

Ventana estándar: 0-3 horas

Ventana extendida: 3-4,5 horas 
Tabla 3

Indicaciones trombólisis IV con rtPA

\begin{tabular}{|l|l|}
\hline \multicolumn{2}{|c|}{ Ventana de tres horas } \\
\hline \multicolumn{1}{|c|}{ Indicación } & Grado de recomendación \\
\hline $\begin{array}{l}\text { Diagnóstico de ACV isquémico } \\
\text { como causa de un déficit } \\
\text { neurológico cuantificable }\end{array}$ & IA \\
\hline $\begin{array}{l}\text { Inicio de los sintomas en }<3 \\
\text { horas }\end{array}$ & IA \\
\hline $\begin{array}{l}\text { Edad } \geq 18 \text { años (igualmente } \\
\text { recomendado para }>\text { y }<\text { de } \\
80 \text { años) }\end{array}$ & IA \\
\hline $\begin{array}{l}\text { ACV isquémico severo (a } \\
\text { pesar de }>\text { riesgo de } \\
\text { transformación hemorrágica, } \\
\text { existe beneficio) }\end{array}$ & IA \\
\hline $\begin{array}{l}\text { ACV menor (sintomas leves, } \\
\text { pero discapacitantes) }\end{array}$ & IB \\
\hline \multicolumn{1}{|c|}{ Indicación } \\
$\begin{array}{l}\text { Diagnóstico de ACV isquémico } \\
\text { Como causa de un déficit } \\
\text { neurológico cuantificable }\end{array}$ & IB \\
\hline $\begin{array}{l}\text { Inicio de los síntomas en } 3- \\
4,5 \text { horas }\end{array}$ & IB \\
\hline Edad $\geq 18$ años Y $\leq 80$ años & IB \\
\hline $\begin{array}{l}\text { Sin historia previa de DM y } \\
\text { ACV previo }\end{array}$ & IB \\
\hline NIHSS $\leq 25$ puntos & IB \\
\hline $\begin{array}{l}\text { No ingesta de anticoagulantes } \\
\text { orales }\end{array}$ & IB \\
\hline $\begin{array}{l}\text { Imagen cerebral sIN evidencia } \\
\text { de compromiso de }>1 / 3 \text { del } \\
\text { territorio de la ACM }\end{array}$ & IB \\
$\begin{array}{l}\text { ACV menor (sintomas leves, } \\
\text { pero discapacitantes) }\end{array}$ & IIB \\
\hline
\end{tabular}

Contraindicaciones para la trombólisis IV con alteplasa

Dentro de las contraindicaciones para la trombólisis IV con alteplasa se encuentran pacientes con tiempo de evolución indeterminado o con tiempo $>4,5$ horas, evidencia de hemorragia intracraneal o evidencia de compromiso isquémico extenso y ya instaurado en la TAC cerebral simple. Así mismo, son contraindicación el haber presentado un ACV isquémico, trauma craneoencefálico severo o cirugía intracraneal/espinal en los últimos 3 meses, o punción arterial de vaso no compresible en los 7 días previos. $\mathrm{El}$ antecedente de neoplasia gastrointestinal (GI) o sangrado GI en los 21 días previos, o tener una malformación vascular intracraneal no rota, no tratada, se considera de alto riesgo y potencialmente peligroso. Dentro de los exámenes complementarios, es contraindicación tener más de 10 microsangrados en la RM cerebral, plaquetas $<100.000 / \mathrm{mm}^{3}$, INR $>$ 1,7, PTT $>40 \mathrm{~s}$, PT $>15 \mathrm{~s}$; también el tratamiento con heparinas de bajo peso molecular a dosis plena en las últimas $24 \mathrm{~h}$, antiplaquetarios inhibidores de la glicoproteína IIb/IIIa, tratamiento con inhibidores directos de la trombina (Dabigatrán) o ihnibidores del factor Xa (Fondaparinux, Rivaroxabán, Apixabán), a menos que tengan pruebas de laboratorio normales o que la última dosis haya sido $>$ $48 \mathrm{~h}$ con función renal normal, entre otras contraindicaciones relativas o absolutas, como lo son la sospecha de endocartitis infecciosa, disección aórtica o la presencia de un trombo cardiaco intracavitario o neoplasia cerebral intraaxial, por el riesgo de sangrado $(32,46)$.

Indicaciones de administración de alteplasa IV para ACV isquémico agudo en ventana (32)

1. Calcular la dosis total de rtPA intravenoso a 0,9 mg/kg (máximo $90 \mathrm{mg}$ de dosis total). Aplicar el 10\% de la dosis en bolo en 1 minuto y el resto en infusión continua en $1 \mathrm{~h}$.

2. Ingresar al paciente en unidad de cuidado intensivo o unidad de stroke para monitoreo.

3. Si el paciente desarrolla cefalea severa, náuseas, vómito, hipertensión aguda o empeoramiento del examen neurológico, se debe suspender la infusión y tomar urgente una TAC cerebral simple.

4. Medir la tensión arterial y realizar examen neurológico así:

a) Cada 15 min durante la infusión, y en las primeras $2 \mathrm{~h}$ posterior a esta.

b) Cada $30 \mathrm{~min}$ durante las siguientes $6 \mathrm{~h}$.

c) Cada $60 \mathrm{~min}$ dentro de las primeras $24 \mathrm{~h}$ de tratamiento.

5. Incremente la frecuencia de medición de la TA si la TAS $>180 \mathrm{~mm}$ $\mathrm{Hg}$ o si la TAD > $105 \mathrm{~mm} \mathrm{Hg}$; administrar medicación antihipertensiva 
para mantener la TA por debajo de estos niveles.

6. Se debe evitar el uso de sonda nasogástrica, sonda vesical o catéter de presión intraarterial si el paciente se puede manejar de forma segura sin ellos.

7. Obtener una TAC cerebral simple de control o RM cerebral a las $24 \mathrm{~h}$ después de la terapia con alteplasa IV, $\mathrm{y}$ antes de iniciar anticoagulantes $\mathrm{o}$ antiplaquetarios.

\section{Complicaciones de la trombolisis intravenosa}

El angioedema orolingual es un efecto adverso al rTPA, alcanzando una incidencia del 1 al 5\%. En caso de presentarse, inicialmente es fundamental asegurar la vía aérea. Si el edema se limita a los labios o la parte anterior de la lengua, la intubación orotraqueal (IOT) puede no ser necesaria; sin embargo, si el edema afecta la laringe, el paladar, el piso de la boca o la orofaringe, con rápida progresión (30 minutos), tiene alto riesgo de requerir IOT. Idealmente, debe hacerse en secuencia rápida con el paciente despierto. Puede requerirse intubación nasal; sin embargo, con riesgo de epistaxis postalteplasa IV. Debe suspenderse la infusión de alteplasa y de inhibidores de la enzima convertidora de angiotensina, en caso de que se estén administrando, así como iniciar manejo con metilprednisolona de $125 \mathrm{mg}$ IV, difenhidramina de $50 \mathrm{mg}$ IV o ranitidina de $50 \mathrm{mg}$ IV. En caso de persistencia del angioedema, administre adrenalina $(0,1 \%) \quad 0,3 \mathrm{~mL}$ subcutáneo o $0,5 \mathrm{~mL}$ nebulizado. El Icatibant, un antagonista selectivo de los receptores de bradiquinina $\mathrm{B} 2$, se usa con éxito en casos de angioedema hereditario y asociado a inhibidores de la enzima convertidora de angiotensina; pero no está disponible en nuestro país $(32,47,48)$.

El sangrado intracerebral sintomático relacionado con la administración de alteplasa IV puede presentarse hasta en el 7\% de los pacientes; y es mucho más baja la presentación $(0,4-0,9 \%)$ cuando se usan trombolíticos con indicación diferente a la de ACV isquémico agudo. Aproximadamente, el $40 \%$ de estos pacientes hace expansión del hematoma, que lleva a una mortalidad de hasta el $61 \%$, a 3 meses.

En caso de existir sospecha de sangrado intracerebral, ya sea por alteración del estado de conciencia, nueva o mayor focalización neurológica, crisis convulsiva durante la infusión, entre otras, debe suspenderse la infusión de alteplasa y trasladar al paciente a toma de TAC cerebral simple de forma inmediata; realizar de manera paralela hemograma, PT, PTT, fibrinógeno y hemoclasificación. En caso de confirmarse el sangrado, se debe iniciar manejo con crioprecipitados de 10 unidades en infusión durante 10 a $30 \mathrm{~min}$, con posibilidad de aplicación de dosis adicionales, en caso de que haya concentraciones de fibrinógeno por debajo de $200 \mathrm{mg} / \mathrm{dL}$. Se continúa con infusión IV de $1 \mathrm{~g}$ de ácido tranexámico en $10 \mathrm{~min}$. Debe ser valorado por hematología y neurocirugía para evaluar medidas adicionales y se debe brindar una terapia general de soporte, que incluye, entre otros, control de tensión arterial, temperatura y glucemia $(32,47,48)$.

\section{Trombólisis intraarterial (32)}

El tratamiento inicial con trombólisis intraarterial es benéfico para pacientes seleccionados con ACV isquémico mayor, < $6 \mathrm{~h}$ de duración, con oclusión de ACM (recomendación grado I).

\section{Trombectomía mecánica (32)}

Pacientes elegibles para trombólisis IV en ventana estándar o extendida deben recibir terapia endovascular si cumplen todos los siguientes criterios (recomendación IA):

Escala Rankin modificada previa a ACV: mRS $0-1$.

Etiología de ACV: oclusión de arteria carótida interna (ACI) o ACM M1.

Escala $\geq 18$ años.

Escala de NIHSS $\geq 6$.

ASPECTS $\geq 6$. 
Tratamiento puede ser iniciado (tiempo aguja) en 6 horas desde el inicio de los síntomas.

Desde los primeros estudios pivotales de trombolisis IV, IA y trombectomía mecánica, se conocen buenas tasas de recanalización con terapias solas y combinadas, como es el caso del Mechanical Embolus Removal in Cerebral Ischemia (MERCI 1), que mostró una recanalización exitosa (TICI 2-3) en el $43 \%$ de los pacientes, y en el $64 \%$ con alteplasa IV adicional. Así mismo, en el PENUMBRA (Penumbra System) y RECOST (Rescue, Combined, and Stand-Alone Thrombectomy in the Management of Large Vessel Occlusion Stroke Using the Solitaire Device) se alcanzaron tasas exitosas de recanalización (TICI 2-3) en el $81,6 \%$ y el $88 \%$ de los pacientes, respectivamente $(49,50,51)$.

Teniendo en cuenta los resultados de los estudios clínicos que emplean stents para la trombectomía mecánica (SWIFT, MR. CLEAN, EXTEND-IA, ESCAPE y REVASCAT), el 29 de junio de 2015 se publicó la actualización de las guías de manejo temprano de pacientes con ACV isquémico agudo con manejo endovascular de la AHA/ASA, concluyendo que la información obtenida en estos estudios permite una recomendación clase I, nivel de evidencia A para la trombectomía mecánica con stent, para un grupo seleccionado de pacientes $(52,53,54,55,56)$.

\section{Manejo médico y prevención secundaria}

\section{Antiplaquetarios}

Tanto en la guía de STROKE 2018 como en la guía de práctica clínica colombiana se recomienda la administración de antiagregantes plaquetarios para el manejo del $\mathrm{ACV}$ isquémico agudo como prevención secundaria. Se debe iniciar esta terapia dentro de las primeras 24-48 $\mathrm{h}$ de iniciados los síntomas; no obstante, en pacientes que recibieron manejo trombolítico con alteplasa se sugiere esperar $24 \mathrm{~h}$ antes de iniciar la terapia antiagregante, aunque es importante evaluar cada caso de manera individual teniendo en cuenta los riesgos y beneficios para iniciar la terapia en el momento más oportuno $(1,32)$.

En cuanto a la antiagregación dual, no hay suficiente evidencia para recomendar su uso rutinario para el tratamiento de pacientes con ACV que no tengan indicación específica para tal (1).

\section{Estatinas}

En un metanálisis de 42 estudios y más de 82.000 pacientes se encontró que el uso de estatinas reduce la incidencia de ACV en hasta el $41 \%$ con atorvastatina (57). Se cree que esto es secundario a la reducción de los niveles séricos de colesterol de baja densidad (LDL) y a los efectos pleiotrópicos de las estatinas, que incluyen efectos antinflamatorios, antioxidantes y neuroprotectores (57). En el estudio SPARCL se demostró que el tratamiento con $80 \mathrm{mg}$ de atorvastatina al día reducía el riesgo de ACV en pacientes sin enfermedad coronaria conocida y LDL entre 100 y $190 \mathrm{mg} / \mathrm{dL}$, quienes hubieran tenido un $\mathrm{ACV}$ o ataque isquémico agudo (AIT) reciente (58). Si no existe contraindicación, se iniciará atorvastatina $80 \mathrm{mg} /$ día dentro de las primeras 24 a 48 h después del ACV (1).

\section{Anticoagulación}

Tanto la guía de STROKE 2018 como la guía de práctica clínica colombiana no recomiendan el uso urgente de anticoagulantes con el objetivo de prevenir recurrencia de ACV, evitar deterioro neurológico o mejorar los desenlaces después de un ACV, ya que no hay evidencia que demuestre que la anticoagulación inmediata se asocie con mayor funcionalidad o disminución de futuros ACV; por el contrario, sí existe un riesgo aumentado de hemorragias intra- o extracraneales que pueden poner en riesgo la vida del paciente $(1,32)$. 


\section{Conclusiones}

El ACV isquémico agudo es una patología con una carga alta de morbilidad en nuestro país y en el resto del mundo, siendo una emergencia neurológica frecuente y la segunda causa de muerte en el mundo después de la enfermedad coronaria. Ello genera costos elevados en su tratamiento y recuperación, y un gran impacto socioeconómico, por lo que es indispensable para el clínico estar actualizado en cuanto a los avances diagnósticos y terapéuticos que han demostrado impactar en términos de calidad de vida y funcionalidad en estos pacientes.

El enfoque ordenado, basado en un algoritmo de atención (figura 6) del ACV isquémico agudo, permite abordar de manera integral y eficaz a estos pacientes, teniendo en cuenta que, basados en la mejor evidencia disponible $\mathrm{y}$ en las guías internacionales, contamos con una ventana de tiempo de hasta 4,5 $\mathrm{h}$ para trombólisis intravenosa y de hasta $6 \mathrm{~h}$ para realizar trombectomía mecánica en pacientes con oclusión de gran vaso y neuroimágenes con evidencia de penumbra isquémica realizando mismatch por resonancia magnética, lo que mejora los desenlaces clínicos y las complicaciones a corto y mediano plazo. Es importante contar con políticas en salud pública enfocadas en educar a la comunidad colombiana en reconocer de manera oportuna los síntomas de un ACV para acudir rápidamente a un centro médico que garantice la atención necesaria.

\section{Figura 6}

Algoritmo de toma de decisiones en el abordaje de un paciente con ACV isquémico agudo

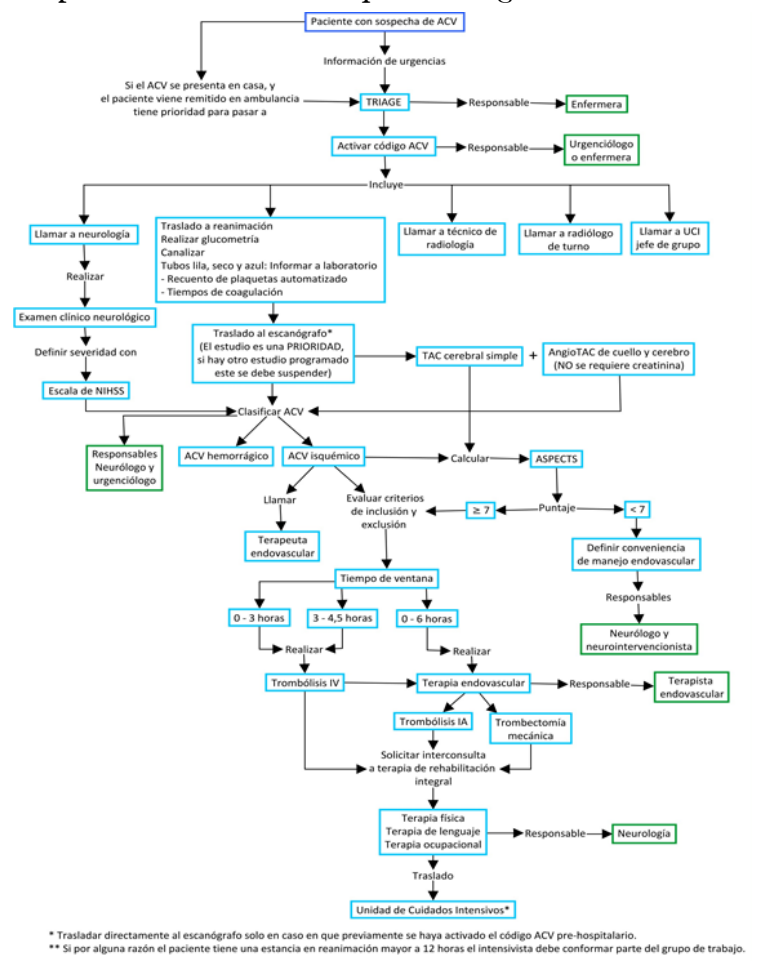

\section{Conflictos de interés}

Los autores declaran no tener ningún conflicto de interés.

\section{Agradecimientos}

Agradecemos al Dr. Javier Francisco Torres, por sus aportes para la realización de este trabajo.

\section{Referencias}

1. Ministerio de Salud y Protección Social. Guía de práctica clínica para el diagnóstico, tratamiento y rehabilitación del episodio agudo del ataque cerebrovascular isquémico en población mayor de 18 años. Vol. 54, CINET. 2015. 1-52. 
2. Arauz A, Ruiz-Franco A. Enfermedad vascular cerebral. Rev Fac Med (Méx.). 2012;55(3):11-21.

3. Mikulik R, Wahlgren N. Treatment of acute stroke: an update. J Intern Med. 2015;278:145-65.

4. Maldonado N, Kazmi S, Suárez J. Update in the management of acute ischemic stroke. Crit Care Clin. 2014;30:673-97.

5. Benjamin EJ, Blaha MJ, Chiuve SE, Cushman M. Heart disease and stroke statistics-2017 update: a report from the American Heart Association. Circulation. 2017;135:146-603.

6. Pradilla AG, Vesga BE, León-Sarmiento FE. Estudio neuroepidemiológico nacional (Epineuro) colombiano. Rev Panam Salud Pública. 2003;14:104-11.

7. Macrez R, Ali C, Toutirais O, et al. Stroke and the immune system: from pathophysiology to new therapeutic strategies. Lancet Neurol. 2011;10:471.

8. Lin MP, Liebeskind DS. Imaging of ischemic stroke. Continuum (NY). 2016;22:1399-423.

9. Jordan JD, Powers WJ. Cerebral Autoregulation and Acute Ischemic Stroke. Am J Hypertens. 2012;25:946-50.

10. Aries MJH, Elting JW, De Keyser J, Kremer BPH, Vroomen PCAJ. Cerebral autoregulation in stroke: a review of transcranial Doppler studies. Stroke. 2010;41:2697-704.

11. Faraci FM. Reactive oxygen species: influence on cerebral vascular tone. J Appl Physiol. 2006;100:739-43.

12. Deb P, Sharma S, Hassan KM. Pathophysiologic mechanisms of acute ischemic stroke: An overview with emphasis on therapeutic significance beyond thrombolysis. Pathophysiology. 2010;17:197.
13. Zarco L, González F, Coral J. Tratamiento actual del ataque cerebrovascular isquémico (ACV) agudo. Univ Méd (Bogotá). 2008;49:467-98.

14. Sierra C, Coca A, Schiffrin EL. Vascular mechanisms in the pathogenesis of stroke. Curr Hypertens Rep. 2011;13:200-7.

15. Lu GW, Liu HY. Downregulation of nitric oxide in the brain of mice during their hypoxic preconditioning. J Appl Physiol. 2001;91:1193-8.

16. Love S. Oxidative stress in brain ischemia. Brain Pathol. 1999;9:119-31.

17. Dugan LL, Choi DW. Excitotoxicity, free radicals, and cell membrane changes. Ann Neurol. 1994;35:S17-21.

18. Brott T, Jr HPA, Olinger CP, Marler JR, Barsan WG, Biller J, et al. Measurements of acute cerebral infarction: a clinical examination scale. Stroke. 1989;20:864-70.

19. Angosto MC. Bases moleculares de la apoptosis. Académica Número la Real Acad Nac Farm. 2003;69:36-64.

20. Friedlander RM. Apoptosis and caspases in neurodegenerative diseases. N Engl J Med. 2003;348:1365-75.

21. Bandera E, Botteri M, Minelli C, Sutton A, Abrams KR, Latronico N. Cerebral blood flow threshold of ischemic penumbra and infarct core in acute ischemic stroke: a systematic review. Stroke. 2006;37:1334-9.

22. Staykov D, Gupta R. Hemicraniectomy in malignant middle cerebral artery infarction. Stroke. 2011;42:513-6.

23. Simard JM, Chen M, Tarasov K V., Bhatta S, Ivanova S, Melnitchenko L, et al. Newly expressed SUR1-regulated NCCa-ATP channel mediates cerebral edema after ischemic stroke. Nat Med. 2006;12:433-40. 
24. Stokum JA, Gerzanich V, Simard JM. Molecular pathophysiology of cerebral edema. J Cereb Blood Flow Metab. 2016;36:513-38.

25. Adams HP, Bendixen BH, Kappelle LJ, Biller J, Love BB, Gordon DL, et al. Classification of subtype of acute ischemic stroke: definitions for use in a multicenter clinical trial. TOAST. Trial of Org 10172 in acute stroke treatment. Stroke. 1993;24:35-41.

26. Alexandru R, Terecoasă EO, Tiu C. Etiologic classification of ischemic stroke: Where do we stand? Clin Neurol Neurosurg. 2017;159:93-106.

27. Molina-Seguin J, Vena $A B$, Colàs-Campàs L, Benalbdelhak I, Purroy F. Revisión sistemática de las características y pronóstico de los sujetos que sufren un ictus criptogénico no lacunar de mecanismo embólico. Neurología. 2018;66:325-30.

28. Pare JR, Kahn JH. Basic neuroanatomy and stroke syndromes. Emerg Med Clin North Am. 2012;30:601-15.

29. Wintermark M, Sanelli PC, Albers GW, Bello JA, Derdeyn CP, Hetts $\mathrm{SW}$, et al. Imaging recommendations for acute stroke and transient ischemic attack patients: a joint statement by the American Society of Neuroradiology, the American College of Radiology and the Society of NeuroInterventional Surgery. J Am Coll Radiol. 2013;20:828-32.

30. Southerland AM. Clinical evaluation of the patient with acute stroke. Continuum. 2017;23:40-61.

31. Birenbaum D, Bancroft LW, Felsberg GJ. Imaging in acute stroke. West J Emerg Med. 2011;12:67-76.

32. Powers WJ, Rabinstein AA, Ackerson T, Adeoye OM, Bambakidis NC, Becker K, et al. 2018 guidelines for the early management of patients with acute ischemic stroke: a guideline for healthcare professionals from the American Heart Association/ American Stroke Association. Stroke. 2018:46-110.

33. Hill M, Rowley HA, Adler F, Eliasziw M, Furlan A, Higashida RT. Selection of acute ischemic stroke patients for intra-arterial thrombolysis with pro-urokinase by using ASPECTS. Stroke. 2003;34:1925-31.

34. Vilela P, Rowley HA. Brain ischemia: CT and MRI techniques in acute ischemic stroke. Eur J Radiol. 2017;96:162-72.

35. Bouchez L, Sztajzel R, Vargas MI, et al. CT imaging selection in acute stroke. Eur J Radiol. 2017;96:153-61.

36. Martinon E, Lefevre PH, Thouant P, Osseby GV, Ricolfi F, Chavent A. Collateral circulation in acute stroke: assessing methods and impact: a literature review. J Neuroradiol. 2014;41:97-107.

37. Hao Y, Yang D, Wang H, Zi W, Zhang M, Geng Y, et al. Predictors for symptomatic intracranial hemorrhage after endovascular treatment of acute ischemic stroke. Stroke. 2017;48:1203-9. https://doi.org/10.116 1/STROKEAHA.116.016368.

38. Havenon A, Mlynash M, KimTenser MA, et al. DEFUSE 3: Good collaterals are associated with reduced ischemic core growth but not neurologic outcome. Stroke. 2019;50(3):632-8.

39. Brozici M, van der Zwan A, Hillen B. Anatomy and functionality of leptomeningeal anastomoses: a review. Stroke. 2003;34:2750-62.

40. Donnan GA, Davis SM. Neuroimaging, the ischaemic penumbra, and selection of patients for acute stroke therapy. Lancet Neurol. 2002;1:417-25. 
41. Nogueira RG, Jadhav AP, Haussen DC, Bonafe A, Budzik RF, Bhuva P, et al. Thrombectomy 6 to 24 Hours after Stroke with a Mismatch between Deficit and Infarct- DAWN. N Engl J Med. 2018; 378:11-21.

42. Albers GW, Marks MP, Kemp S, Christensen S, Tsai JP, OrtegaGutiérrez S, et al. Thrombectomy for stroke at 6 to 16 hours with selection by perfusion imaging-DEFUSE 3. N Engl J Med. 2018;708-18.

43. Srinivasan A, Goyal M, al Azri F, Lum C. State-of-the-art imaging of acute stroke. RadioGraphics. 2006;26:S75-95.

44. Cockroft KM, Prestigiacomo CJ, Duffis EJ, Gandhi CD. Cerebral angiography: surgical endovascular neuroradiology. New York: Thieme; 2015.

45. Jayaraman MV, Grossberg JA, Meisel KM, Shaikhouni A, Silver B. The clinical and radiographic importance of distinguishing partial from near-complete reperfusion following intra-arterial stroke therapy. Am J Neuroradiol. 2013;34:135-9.

46. Powers WJ, Derdeyn CP, Biller J, Coffey CS, Hoh BL, Jauch EC, et al. 2015 American Heart Association/ American Stroke Association Focused Update of the 2013 Guidelines for the Early Management of Patients with Acute Ischemic Stroke Regarding Endovascular Treatment. Stroke. 2015;46:3020-35.

47. Pahs L, Droege C, Kneale $\mathrm{H}$, Pancioli A. A novel approach to the treatment of orolingual angioedema after tissue plasminogen activator administration. Ann Emerg Med. 2016;68:345-8.

48. Frontera JA, Lewin JJ 3rd, Rabinstein AA, Aisiku IP, Alexandrov AW, Cook AM, et al. Guideline for reversal of antithrombotics in intracranial hemorrhage: a statement for healthcare professionals from the Neurocritical Care Society and Society of Critical Care Medicine. Neurocrit Care. 2016;24:6-46.

49. Gobin YP, Starkman S, Duckwiler GR, Grobelny T, Kidwell CS, Jahan $\mathrm{R}$, et al. MERCI 1: A phase 1 study of mechanical embolus removal in cerebral ischemia. Stroke. 2004;35:2848-53.

50. Po Sit S, Langer D, Stingele R. The penumbra pivotal stroke trial: Safety and effectiveness of a new generation of mechanical devices for clot removal in intracranial large vessel occlusive disease. Stroke. 2009;40:2761-8.

51. Costalat V, MacHi P, Lobotesis K, Maldonado I, Vendrell JF, Riquelme $\mathrm{C}$, et al. Rescue, combined, and stand-alone thrombectomy in the management of large vessel occlusion stroke using the solitaire device: A prospective 50-patient single-center study: Timing, safety, and efficacyRECOST. Stroke. 2011;42:1929-35.

52. Saver JL, Jahan R, Levy EI, Jovin TG, Baxter B, Nogueira RG, et al. Solitaire flow restoration device versus the Merci Retriever in patients with acute ischaemic stroke (SWIFT): A randomised, parallelgroup, non-inferiority trial. Lancet. 2012;380:1241-9.

53. Berkhemer O, Fransen PS, Beumer D, Lingsma H. A Randomized trial of intraarterial treatment for acute ischemic stroke - MR CLEAN. N Engl J Med. 2015;372:2747-57.

54. Campbell BCV, Mitchell PJ, Kleinig TJ, Dewey HM, Churilov L, Yassi $\mathrm{N}$, et al. Endovascular therapy for ischemic stroke with perfusion-imaging selection-EXTEND-IA. N Engl J Med. 2015;372:1009-18. 
55. Demchuk AM, Goyal M, Menon BK, Eesa M, Ryckborst KJ, Kamal $\mathrm{N}$, et al. Endovascular treatment for small core and anterior circulation proximal occlusion with emphasis on minimizing $\mathrm{CT}$ to recanalization times (ESCAPE) trial: methodology. Int J Stroke. 2015;10:429-38.

56. Molina CA, Chamorro A, Rovira À, de Miquel A, Serena J, Roman LS, et al. REVASCAT: A randomized trial of revascularization with SOLITAIRE

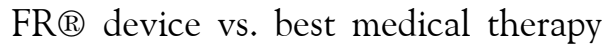
in the treatment of acute stroke due to anterior circulation large vessel occlusion presenting within eighthours of symptom onset. Int J Stroke. 2015;10:619-26.

57. Guzik A, Bushnell C. Stroke epidemiology and risk factor management. Continuum (Minneap Minn). 2017;23:15-39.

58. Amarenco P, Bogousslavsky J, Callahan A, et al. High-dose atorvastatin after stroke or transient ischemic attack. $N$ Engl J Med. 2006;355:549-59. 